\title{
On the Accuracy of Spectral Element Method in Electromagnetic Scattering Problems
}

\author{
I. Mahariq, H. I. Tarman, and M. Kuzuoğlu
}

\begin{abstract}
Spectral element method (SEM), which is known of its high accuracy, has been recently applied in solving electromagnetic problems governed by Maxwell's equations. This paper investigates the accuracy of SEM in twodimensional, frequency-domain electromagnetic scattering problems where Helmholtz equation acts as the governing partial differential equation (PDE). As experience in meshing a problem in finite element method is important to obtain accurate results, the choice of elements in SEM, on the other hand, is important too. The aspect ratio in this paper is taken into account while studying the accuracy in a single element by utilizing the Green's function. In addition, the scalar field scattered by a circular cylinder placed in front of an incident plane wave is solved after truncating the domain by perfectly matched layer. Numerical results show that one should carefully discretize the problem and keeping the aspect ratio close to unity as much as possible to guarantee accurate results.
\end{abstract}

Index Terms-Aspect ratio, electromagnetic scattering, PML SEM.

\section{INTRODUCTION}

It is more an art experience than a science to know how to optimally place and size the mesh in finite element method (FEM). In fact, experience taught us to have more elements in the physical domain where functions change rapidly and less elements where low gradient is expected. Mesh generation may take several trials before achieving a good mesh [1]. On the other hand, the complexity in the physical domain itself may add additional limitations to mesh generation.

In FEM, ranges of the aspect ratio have been investigated extensively and for wide variety of problems. As an example, but not restricted to, M. Picasso [2] proposed an adaptive algorithm for solving the Strokes problem with finite elements and meshes with high aspect ratio. In that paper, the effect of aspect ratio on the results is discussed in details and some examples were illustrated for a non-acceptable mesh that can deteriorate accuracy. V. Prachittham et al. [3] presented a two-dimensional adaptive method with large aspect ratio finite elements for the numerical simulation of mixed electroosmotic microflows. In their work, the refinement/ coarsening criterion is based on a posteriori error estimates. On the other side, spectral element method (SEM) which is known for its high degree of accuracy and lower CPU time and less memory requirement, when compared

Manuscript received December 20, 2013; revised March 20, 2014.

I. Mahariq is with Middle East Technical University, Turkey (e-mail: ibmahariq@gmail.com).

H. I. Tarman is with the Dept. of Engineering Sciences in Middle East Technical University, Ankara, Turkey (e-mail: tarman@metu.edu.tr).

M. Kuzuoğlu is with the Dept. of Electrical and Electronics Eng. in Middle East Technical University (e-mail: kuzuoglu@ metu.edu.tr). with other numerical methods, has the flexibility of using larger elemental aspect ratio without significant deterioration in accuracy. S. Dong et al. [4] proposed a parallel SEM for dynamic three-dimensional nonlinear elasticity problems that provides a tolerant large elemental aspect ratio employing Jacobi polynomial-based shape functions, as an alternative to the typical Legendre polynomial-based shape functions in solid mechanics. D. Rh. Gwynllyw et al. [5] proposed an iterative method for moving SEM applied to the journal bearing problem where they investigated the results of extremely large physical aspect ratio.

Recently, the features of SEM have attracted researchers to utilize this method in electromagnetic problems [6]-[8]. However, the effect of elemental aspect ratio on accuracy has not been investigated in numerical modeling of electromagnetic radiation and/or scattering problems when SEM is utilized. Thus, the motivation of this work is to investigate how much deterioration in solution accuracy is obtained at different values of elemental aspect ratio in two-dimensional electromagnetic scattering problems. We confirm that no such work exists in the literature. This kind of investigation is very important in the sense that one can depend on this work when deciding how to accurately mesh the physical domain using SEM. In contrast to FEM, elements or subdomains in SEM has LegendreGauss-Lobatto grids, i.e., once the elements are chosen, its mesh can't be changed. This study utilizes Legendre polynomials as the basis functions. Finally, to check how results obtained from single-element problems are related to typical electro- magnetic scattering problems in which the well-known Perfectly Matched Layer (PML) is utilized for domain truncation [9].

The content of this paper is arranged as follows: in Section II, the equations to be solved using SEM are presented. In Section III, shapes of the elements in SEM are discussed. Section IV demonstrates the numerical results and discussion, and finally some conclusions are presented in Section V.

\section{Electromagnetic ScAtTERING Problems}

Electromagnetic scattering problems in two-dimensional free-space regions are reduced to the two-dimensional homogenous Helmholtz equation:

$$
\frac{\partial^{2} u}{\partial x^{2}}+\frac{\partial^{2} u}{\partial y^{2}}+k^{2} u=0
$$

where $u(x, y)$ is the scalar field to be solved, and $k$ is the wave number defined as: 


$$
k=\frac{2 \pi}{\lambda}
$$

with $\lambda$ being the wavelength. In a typical, unbounded electromagnetic scattering problem, the domain is truncated by an absorbing boundary layer or the so-called Perfectly Matched Layer (PML) which was introduced by J. P. Berenger [9]. Fig. 1 shows the scatterer in the free space region $\left(\Omega_{F S}\right)$ surrounded by the $\operatorname{PML}\left(\Omega_{P M L}\right)$.

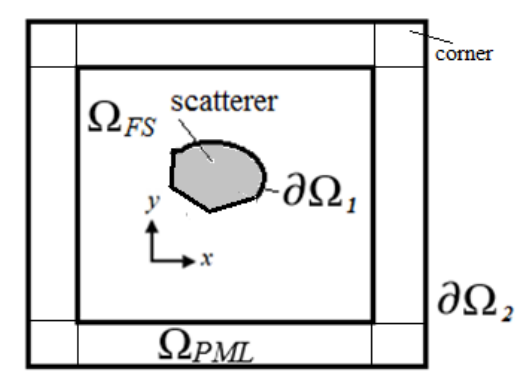

Fig. 1. A typical electromagnetic computational domain

The PML formulation in [10] is adopted in this work. That is, attenuating the scalar field along $x$-direction in the PML region is governed by the non-homogenous Helmholtz equation:

$$
\frac{1}{a^{2}} \frac{\partial^{2} u}{\partial x^{2}}+\frac{\partial^{2} u}{\partial y^{2}}+k^{2} u=0
$$

where $a$ is defined as:

$$
a=1+\frac{\alpha}{j k}
$$

and $a$ is a positive real constant (called attenuation factor). Similarly, attenuating the scalar field along $y$-direction in the PML region is governed by:

$$
\frac{\partial^{2} u}{\partial x^{2}}+\frac{1}{a^{2}} \frac{\partial^{2} u}{\partial y^{2}}+k^{2} u=0
$$

And wherever there is a corner in the PML region, attenuation is applied in both directions, i.e., the following equation must be satisfied:

$$
\frac{1}{a^{2}} \frac{\partial^{2} u}{\partial x^{2}}+\frac{1}{a^{2}} \frac{\partial^{2} u}{\partial y^{2}}+k^{2} u=0
$$

Equations (1) to (6) can be expressed as follows:

$$
\nabla . \Lambda \nabla u+a k^{2} u=0
$$

in which the tensor $\Lambda$ is defined as:

$$
\Lambda=\left[\begin{array}{cc}
\Lambda_{11} & 0 \\
0 & \Lambda_{22}
\end{array}\right]
$$

where;

$$
\begin{gathered}
{\left[\begin{array}{ll}
\Lambda_{11} & \Lambda_{22}
\end{array}\right]=\left[\begin{array}{ll}
\frac{1}{a} & a
\end{array}\right] \text { for } x \text {-decay, }} \\
{\left[\begin{array}{ll}
\Lambda_{11} & \Lambda_{22}
\end{array}\right]=\left[\begin{array}{ll}
a & \frac{1}{a}
\end{array}\right] \text { for } y \text {-decay, }} \\
{\left[\begin{array}{ll}
\Lambda_{11} & \Lambda_{22}
\end{array}\right]=\left[\begin{array}{ll}
\frac{1}{a} & \frac{1}{a}
\end{array}\right] \text { for a corner region, }}
\end{gathered}
$$

and $a=1$ for $\Omega_{F S}$.

The numerical implementation of SEM introduced in [8] is adopted in this paper with Legendre polynomials being the basis functions.

\section{SHAPES OF 2D-ELEMETS}

Lagrangian basis polynomials associated with a tensor product grid of Gauss-Lobbatto-Legendre (GLL) nodes is used to build a nodal basis for a reference element having dimensions $[-1,1] \times[-1,1]$. Fig. 2 illustrates an example of such a grid for a tenth-order polynomial space (nodes are represented by intersections between horizontal and vertical lines).

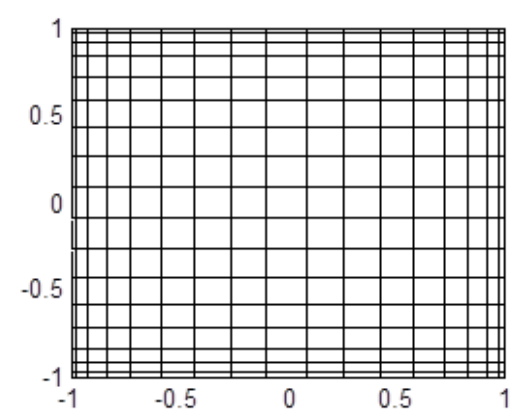

Fig. 2. GLL grid nodes on the reference element.

In contrast to FEM where two dimensional element can be chosen to be triangular, in SEM, quadrilateral elements are the only possible elements. Fortunately, quadrilateral elements with straight sides or curved sides are applicable to SEM (See Fig. 3).
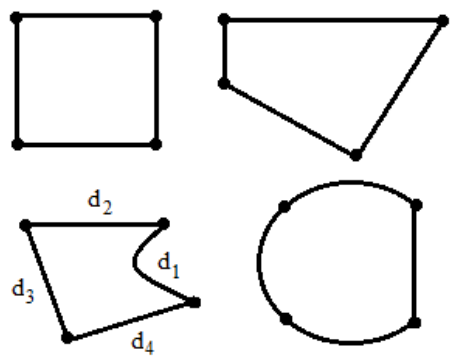

Fig. 3. Various quadrilateral elements that are applicable to SEM.

In this work, the following definition for the elemental aspect ratio $(A R)$ is considered:

$$
A R=\frac{\max \left(d_{i}\right)}{\min \left(d_{i}\right)}
$$


where $d_{i}$ stands for side length of an element, $i=1,2,3,4$. To make use of this definition, one needs to study the accuracy of SEM for a single-element problem having a reference area with equal dimensions. Then, by changing the dimensions of the element while having the same area, a comparison can be performed. With this approach, the effect of $A R$ on the accuracy can be well investigated. It is also worth here to point that all sides of the elements are assumed to have equal nodes.

\section{NUMERICAL RESUltS AND DisCUSSION}

The two-dimensional Green's function that has Helmholtz equation as the governing PDE (point source problem):

$$
\nabla^{2} u+k^{2} u=-\delta(\vec{r})
$$

is considered in our study. To avoid singularity in the solution, the point source is placed at the origin of the $x y$-plane so that the homogenous Helmholtz equation is to be solved inside a square element $(\Omega)$ with dimensions $\lambda \times \lambda$, and $\lambda=1$; hence the element has a unit area (See Fig. 4). This square element will be referred as the reference element. On the boundary $\partial \Omega$ the exact solution in terms of Hankel function of the second kind (zero order), $\left(u(\vec{r})=(j / 4) H_{0}^{(2)}(k|\vec{r}|)\right.$, is applied.

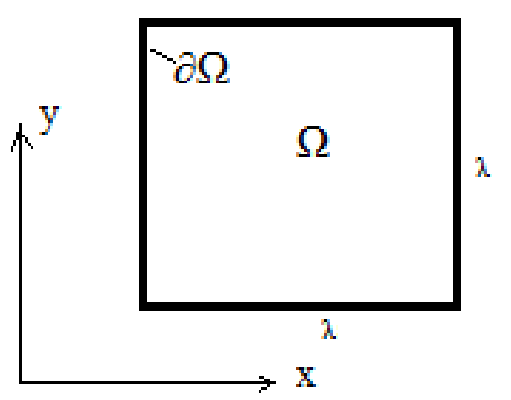

Fig. 4. The reference square element with unit area.

The real part of the solution is shown in Fig. 5 for $N=18$ points per wavelength. Throughout this work, the maximum relative error is defined as:

$$
E r r=\max _{i} \frac{\left|u_{i, \text { exact }}-u_{i, S E M}\right|}{\left|u_{i, \text { exact }}\right|}
$$

where $u_{i, \text { exact }}$ and $u_{i, S E M}$ are the exact solution and the SEM solution, respectively, at the $i^{\text {th }}$ node corresponding to the free space region, $\Omega$. The error is presented in Table I as $N$ increases.

Now we consider a quadrilateral element with straight sides and having a unit area as shown in Fig. 6. The error is presented in Table II for the aspect ratios $A R=1.33,1.88$ and 2.87 (while the elemental area is kept the same). In Table III, the errors are represented in a normalized form with respect to the errors obtained from the reference element. It is very obvious to observe that as the aspect ratio $(A R)$ increases, the accuracy is highly deteriorated. But one wonders whether this deterioration occurs for other types of element.

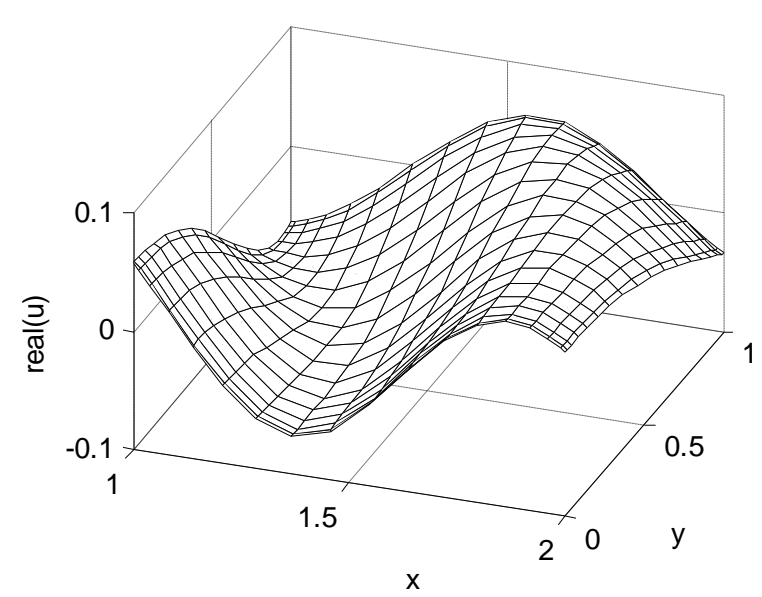

Fig. 5. Real part of the solution in the reference element at $N=18$.

TABLE I: ERRORS VS $N$ FOR THE REFERENCE ELEMENT

\begin{tabular}{cc}
\hline \hline $\boldsymbol{N}$ & $\boldsymbol{E r r}$ \\
\hline 7 & 0.00091 \\
8 & $1.30 \mathrm{E}-04$ \\
9 & $1.25 \mathrm{E}-05$ \\
10 & $1.14 \mathrm{E}-06$ \\
11 & $1.13 \mathrm{E}-07$ \\
12 & $1.26 \mathrm{E}-08$ \\
13 & $1.51 \mathrm{E}-09$ \\
14 & $2.23 \mathrm{E}-10$ \\
15 & $2.57 \mathrm{E}-11$ \\
16 & $3.23 \mathrm{E}-12$ \\
17 & $2.86 \mathrm{E}-13$ \\
\hline \hline
\end{tabular}

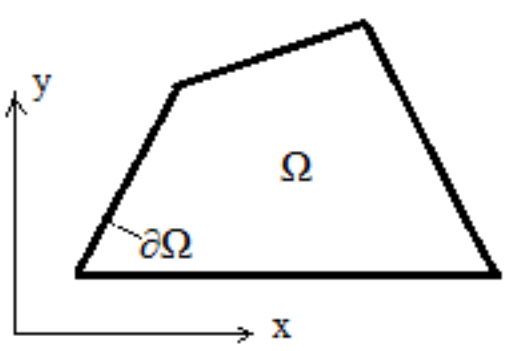

Fig. 6. Quadrilateral element with straight sides and unit area.

TABLE II: ERRORS FOR QUADRILATERAL ELEMENT WITH STRAIGHT SIDES

\begin{tabular}{cccc}
\multicolumn{4}{c}{ AND UNIT AREA } \\
\hline \hline $\mathbf{N}$ & $\boldsymbol{E r r}$ & $\boldsymbol{E r r}$ & $\boldsymbol{E r r}$ \\
& $(\boldsymbol{A} \boldsymbol{R}=$ & $(\boldsymbol{A} \boldsymbol{R}=$ & $(\boldsymbol{A R}=$ \\
& $\mathbf{1 . 3 3})$ & $\mathbf{1 . 8 8})$ & $\mathbf{2 . 8 7 )}$ \\
\hline 7 & 0.001 & 0.003 & 0.062 \\
8 & $1.5 \mathrm{e}-04$ & $6.0 \mathrm{e}-04$ & 0.012 \\
9 & $1.5 \mathrm{e}-05$ & $1.2 \mathrm{e}-04$ & 0.004 \\
10 & $1.6 \mathrm{e}-06$ & $2.4 \mathrm{e}-05$ & 0.001 \\
11 & $1.7 \mathrm{e}-07$ & $4.6 \mathrm{e}-06$ & $2.6 \mathrm{e}-04$ \\
12 & $1.8 \mathrm{e}-08$ & $8.2 \mathrm{e}-07$ & $6.3 \mathrm{e}-05$ \\
13 & $2.0 \mathrm{e}-09$ & $1.4 \mathrm{e}-07$ & $1.5 \mathrm{e}-05$ \\
14 & $2.6 \mathrm{e}-10$ & $2.3 \mathrm{e}-08$ & $3.4 \mathrm{e}-06$ \\
15 & $3.1 \mathrm{e}-11$ & $3.6 \mathrm{e}-09$ & $7.3 \mathrm{e}-07$ \\
16 & $3.8 \mathrm{e}-12$ & $5.5 \mathrm{e}-10$ & $1.5 \mathrm{e}-07$ \\
17 & $4.3 \mathrm{e}-13$ & $8.0 \mathrm{e}-11$ & $2.9 \mathrm{e}-08$ \\
\hline \hline
\end{tabular}

Next, a quadrilateral element having one curved side and a unit area is investigated (See Fig. 7). This kind of elements is encountered in meshing of many computational electromagnetic problems. The solution of the Green's function problem is applied on the boundary of the element where the homogeneous Helmholtz equation is satisfied. The maximum relative errors are presented in Table IV for 
different values of aspect ratio while keeping the elemental area unchanged.

TABLE III: NORMALIZED ERRORS FOR QUADRILATERAL ELEMENT WITH STRAIGHT SidES AND UNIT AREA WITH RESPECT TO REFERENCE ELEMENT

\begin{tabular}{ccrr}
\hline \hline $\boldsymbol{N}$ & $\begin{array}{c}\boldsymbol{E r r} \\
(\boldsymbol{A R}=\end{array}$ & $\begin{array}{c}\boldsymbol{E r r} \\
(\boldsymbol{A R}=\end{array}$ & $\begin{array}{c}\text { Err } \\
(\boldsymbol{A R}=\end{array}$ \\
& $\mathbf{1 . 3 3})$ & $\mathbf{1 . 8 8})$ & \multicolumn{2}{c}{$\mathbf{2 . 8 7})$} \\
\hline 7 & 1.10 & 3.3 & 68 \\
8 & 1.15 & 4.6 & 92 \\
9 & 1.20 & 9.6 & 321 \\
10 & 1.41 & 21.1 & 881 \\
11 & 1.50 & 40.6 & 2296 \\
12 & 1.43 & 65.1 & 5003 \\
13 & 1.32 & 92.5 & 9910 \\
14 & 1.17 & 103.2 & 15259 \\
15 & 1.21 & 140.3 & 28445 \\
16 & 1.18 & 170.5 & 46490 \\
17 & 1.51 & 280.0 & 101501 \\
\hline \hline
\end{tabular}

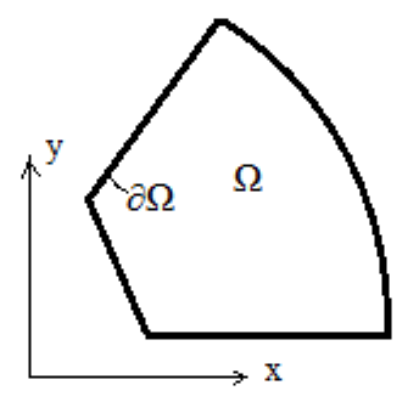

Fig. 7. Quadrilateral element having one curved side and a unit area.

TABLE IV: ERRORS FOR QUADRILATERAL ELEMENT WITH CURVED SIDE

\begin{tabular}{|c|c|c|c|}
\hline$N$ & $\begin{array}{c}\text { Err } \\
(A R= \\
1.39)\end{array}$ & $\begin{array}{c}E r r \\
(A R= \\
1.91)\end{array}$ & $\begin{array}{c}E r r \\
(A R= \\
2.39)\end{array}$ \\
\hline 7 & 0.0014 & 0.0016 & 0.0189 \\
\hline 8 & 0.0005 & 0.0008 & 0.0029 \\
\hline 9 & $1.8 \mathrm{e}-05$ & $3.3 e-05$ & $2.7 \mathrm{e}-04$ \\
\hline 10 & $2.1 \mathrm{e}-05$ & $2.8 \mathrm{e}-05$ & $3.8 \mathrm{e}-05$ \\
\hline 11 & $2.7 \mathrm{e}-07$ & $6.5 \mathrm{e}-07$ & $5.3 \mathrm{e}-06$ \\
\hline 12 & $1.0 \mathrm{e}-07$ & $1.7 \mathrm{e}-07$ & $7.2 \mathrm{e}-07$ \\
\hline 13 & $4.4 \mathrm{e}-08$ & $4.7 \mathrm{e}-08$ & $9.2 \mathrm{e}-08$ \\
\hline 14 & 5.0e-09 & $1.3 \mathrm{e}-08$ & $3.1 \mathrm{e}-08$ \\
\hline 15 & $5.3 \mathrm{e}-10$ & $1.5 \mathrm{e}-09$ & $3.2 \mathrm{e}-09$ \\
\hline 16 & $5.3 \mathrm{e}-11$ & $2.5 \mathrm{e}-10$ & $8.8 \mathrm{e}-10$ \\
\hline 17 & $5.2 \mathrm{e}-12$ & $1.6 \mathrm{e}-10$ & $9.1 \mathrm{e}-10$ \\
\hline
\end{tabular}

Finally, scattering by a circular cylinder is considered. As shown in Fig. 8, the analytical solution of the scattered field due to an incident plane wave $\left(u_{z}^{i}=e^{-j k x}\right)$ on an infinitely long, perfectly conducting cylinder is expressed in terms of Bessel and Hankel functions as:

$$
u^{s}=-u_{0} \sum_{n=-\infty}^{\infty}(-j)^{n} \frac{J_{n}\left(k r_{c}\right) H_{n}^{(2)}(\mathrm{k} \rho) e^{j n \varphi}}{H_{n}^{(2)}\left(k r_{c}\right)}
$$

Because of symmetry in $z$ direction, the problem is a two-dimensional one. And due to symmetry around $x$-axis, only one-half of the plane is studied and symmetric Neumann boundary condition is applied along $x$-axis. The PML region, which is formed by elements $5-10$, is used to truncate the computational domain. Zero-Dirichlet boundary condition is applied on the outer boundary of the PML region, and on the circular boundary, the analytical solution is introduced. Results corresponding to region of interest (elements: 1 to 4) are presented in Table $\mathrm{V}$ for the following dimensions defined on Fig. 9: cylinder radius $r=(0.2,0.5), a=0.5$, $b=c=d=1$ and $\lambda=1$. Real part of the scattered field in the region of interest at $r=0.2, N=17$ is plotted in Fig. 10. It can be clearly seen that as $r$ decreases (AR increases), the error increases sharply. In addition, as the resolution or grid points are increased when high elemental aspect ratio is involved, the rate of error decay is noticeably decreased.

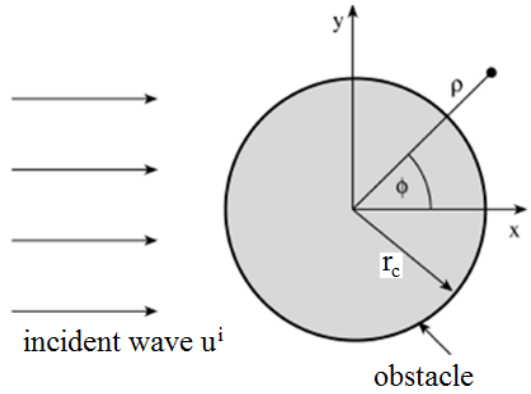

Fig. 8. Scattering cylinder

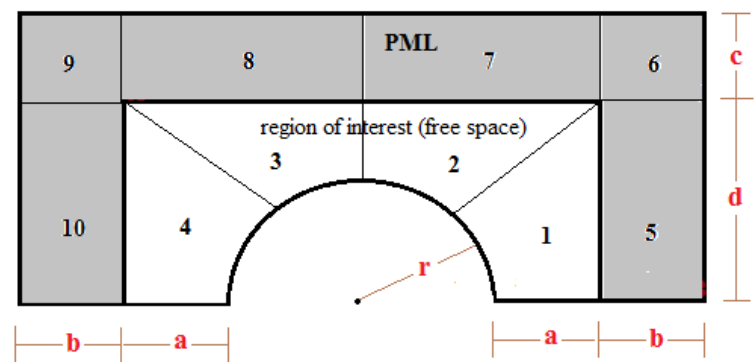

Fig. 9. Elements and dimensions of scattering cylinder problem.

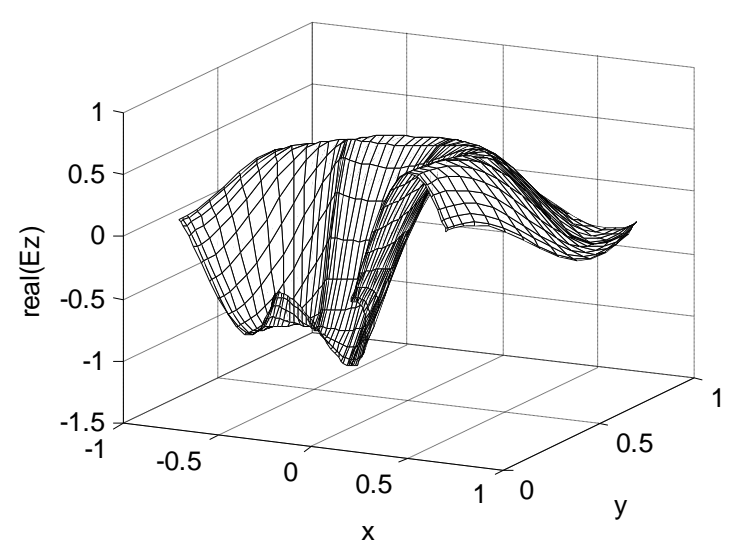

Fig. 10. Real part of the scattered field in the region of interest at $r=0.2, N=$ 17.

TABLE V: ERRORS OF PROBLEM IN FIG. 7.

\begin{tabular}{ccc}
\hline \hline$N$ (points) & $\begin{array}{c}\text { Err } \\
(r=0.5)\end{array}$ & $\begin{array}{c}\text { Err } \\
(r=0.2)\end{array}$ \\
\hline 7 & 0.001994 & 0.00619 \\
8 & 0.000342 & 0.00186 \\
9 & $6.03 \mathrm{E}-05$ & $4.63 \mathrm{e}-4$ \\
10 & $7.77 \mathrm{E}-06$ & $2.17 \mathrm{e}-4$ \\
11 & $9.72 \mathrm{E}-07$ & $1.11 \mathrm{e}-4$ \\
12 & $1.30 \mathrm{E}-07$ & $5.17 \mathrm{E}-05$ \\
13 & $1.50 \mathrm{E}-08$ & $2.37 \mathrm{E}-05$ \\
14 & $1.60 \mathrm{E}-09$ & $1.09 \mathrm{E}-05$ \\
15 & $7.89 \mathrm{E}-10$ & $5.02 \mathrm{E}-06$ \\
16 & $4.65 \mathrm{E}-10$ & $2.32 \mathrm{E}-06$ \\
17 & $3.63 \mathrm{E}-10$ & $1.08 \mathrm{E}-06$ \\
\hline \hline
\end{tabular}




\section{CONCLUSION}

In this paper, the maximum relative error is computed by SEM with Legendre polynomials being the bases functions. The Green's function is used to study the accuracy on single elements at different aspect ratios. And then a real electromagnetic scattering problem is solved. As observed from the results, in elements with one side being curved, the error was less than that of elements having straight sides as the aspect ratio increase. However, in the scattering problem, the accuracy is highly deteriorated when the aspect ratio increases since there are mixed elements of straight and curved sides.

Hence, one can notice from the presented errors that there is no safe range of the aspect ratio in which high accuracy is guaranteed. In conclusion, the discretization of the physical domain should be performed so that the aspect ratio of each element is close to unity as much as possible.

\section{REFERENCES}

[1] D. W. Pepper and J. C. Heinrich, Finite Element Methods: Basic Concepts and Applications, 1st ed. USA: CRC Press, 1992, ch. 4, pp. 56.

[2] M. Picasso, "An adaptive algorithm for the Stokes problem using continuous, piecewise linear stabilized finite elements and meshes with high aspect ratio," Applied Numerical Mathematics, vol. 54, pp. 470-490, August 2005

[3] V. Prachittham1, M. Picasso, and M. Gijs, "Adaptive finite elements with large aspect ratio for mass transport in electroosmosis and pressure-driven microflows," International Journal of Numerical Methods in Fluids, vol. 63, pp. 1005-1030, 2010.

[4] S. Dong and Z. Yosibash, "A parallel spectral element method for dynamic three-dimensional nonlinear elasticity problems," Journal of Computers and Structures, vol. 87, pp. 59-72, 2009.

[5] D. R. Gwynllyw and T. N. Phillips, "Iterative methods with dynamic preconditioning for moving spectral element technique applied to the journal bearing problem," in Proc. the Third International Conference on Spectral and High Order Methods, 1996, pp. 277-286.

[6] J. Lee, T. Xiao, and Q. H. Liu, "A 3-D spectral-element method using mixed-order curl conforming vector basis functions for electromagnetic fields," IEEE Trans. on Microwave Theory and Techniques, vol. 54, no. 1, pp. 437-444, January 2006.

[7] J. Lee and Q. H. Liu, "A 3-D spectral-element time-domain method for electromagnetic simulation," IEEE Trans. on Microwave Theory and Technique, vol. 55, no. 5, pp. 983-991, May 2007.

[8] O. Z. Mehdizadeh and M. Paraschivoiu, "Investigation of a two-dimensional spectral element method for Helmholtz_s equation," Journal of Computational Physics, vol. 189, pp. 111-129, 2003.

[9] J. P. Berenger, "A Perfectly Matched Layer for the Absorption of Electromagnetic Waves," Journal of Computational Physics, vol. 114, pp. 185-200, 1994.

[10] H. Werner and R. Mittra, Frontiers in Electromagnetics, Douglas, IEEE Press, 1999, ch. 14, pp. 609-643.

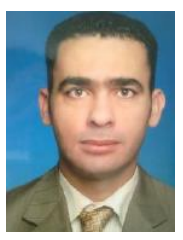

Ibrahim Mahariq took his B.Sc. degree from the Department of Electrical and Computer Engineering, Palestine Polytechnic University, Palestine in 2003. He worked there as a teaching assistant from 2003 to 2005 . He took M.Sc. from Middle East Technical University (METU) in electrical machines and power electronics/ Department of Electrical and Electronics Engineering, Ankara, Turkey. He is currently a PhD student working on computational electromagnetics and nano-photonics application.

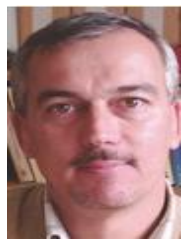

Hakan I. Tarman was born in İzmir, Turkey. He received the B.Sc. degree in mechanical engineering and mathematics, and the M.Sc. degree in mechanical engineering from Boğaziçi University, İstanbul, Turkey. $\mathrm{He}$ received the Ph.D. degree in applied mathematics from Brown University, Providence, Rhode Island, USA. He is currently with the Department of Ankara, Turkey Engineering Sciences, Middle East Technical University,

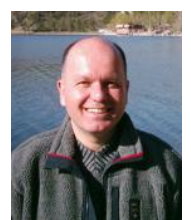

Mustafa Kuzuoglu received the B.Sc., M.Sc., and Ph.D. degrees in electrical engineering from Middle East Technical University (METU), Ankara, Turkey, in 1979 , 1981 , and 1986, respectively. He is currently a professor in METU. His research interests include computational electromagnetics, inverse problems, and radars. 